\title{
ON THE CABLE CAR FEED SUPPORT CONFIGURATION FOR FAST
}

\author{
REN GEXUE ${ }^{1}$, LU QIUHAI and ZHOU ZHOU \\ ${ }^{1}$ Dept. of Engineering Mechanics, Tsinghua University, Beijing, 100084, China \\ E-mail: rengx@mail.tsinghua.edu.cn \\ ${ }^{2}$ Beijing Astronomical Observatory, The Chinese Academy of Sciences Beijing 100080, China
}

\begin{abstract}
This paper introduces the cable car feed support configuration for China's FAST project. Recent advances on the mechanics of the proposed supporting structure and the control of the Stewart platform for secondary feed stabilization are presented. Difficulties associated with the configuration are also discussed.
\end{abstract}

\section{Introduction}

The platformless feed support configuration for large radio telescopes, proposed by Duan et al. (1996), has the potential for dramatically reducing construction cost, provided the required positioning and pointing precision can be guaranteed. The platformless supporting cable structure is less pre-tensioned, because much less weight is supported, than in a platform support structure such as used at Arecibo Observatory. Aiming to increase the stiffness of the platformless supporting system, the first author proposed a cable-car feed support configuration for China's FAST project (Nan et al., 2000). This paper introduces this support configuration, its characteristics and the vibration reduction effects of the pre-tension cables introduced in the configuration. The Stewart platform used as a feed stabilizer is simulated through finite element modelling, with a control scheme based on a finite-difference prediction proposed by the authors.



Figure I. The cable car support configuration. 


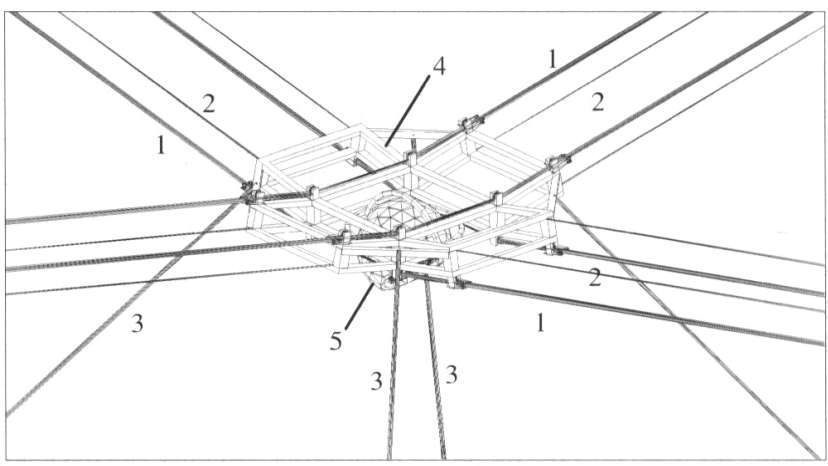

Figure 2. The cable car and the feed cabin.

1: suspension cable; 2 : driving cable; 3 : pre-tension cable; 4: cable car; 5 : feed cabin.

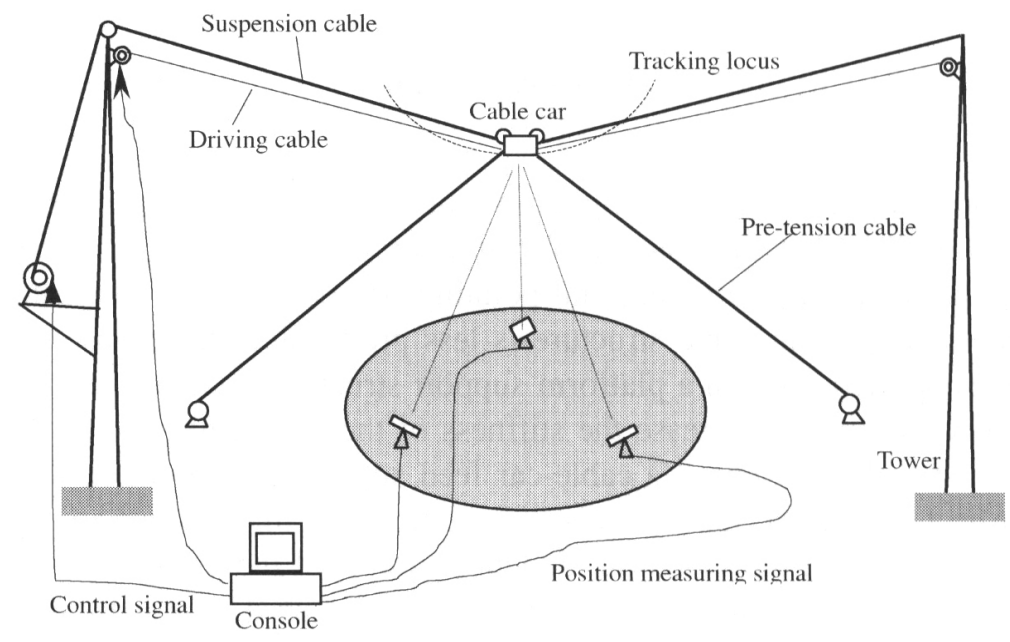

Figure 3. The general assembly of the cable car feed support configuration.

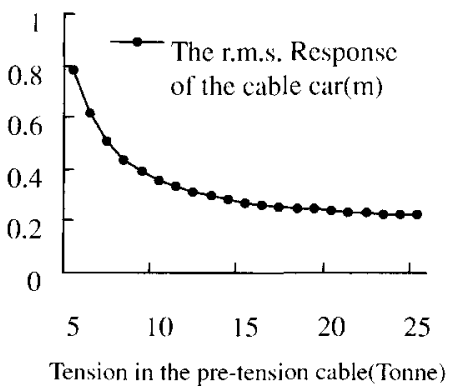

Figure 4. The effects of pre-tension on the responses. 


\section{The Cable Car feed Supporting Configuration for FAST}

The cable-car feed support configuration consists of two crosswise sets of cables suspended on two pairs of opposite towers, a cable car that can move in two directions with the two sets of suspension cables as tracks, and four downward pre-tensioning cables, as shown in Figure 1. The receivers are mounted on a Stewart platform held by six actuators in the feed cabin. The cabin has two rotational degrees of freedom relative to the cable car, which allows the feed to be arbitrarily pointed, irrespective of what the orientation of the cable car is, see Figure 2. For the cable car to track astronomical loci, the lengths of two sets of the suspension cables and the driving cables of the cable car have to be adjusted appropriately. One end of the four pre-tensioning cables is fixed on, and passively moves with, the cable car; the other end is constantly loaded at the anchorage position, slightly offset above the reflecting surface. The configuration is easier to understand by referring to the two-dimension model in Figure 3. The pre-tension cables are introduced for adjusting the stiffness of the feed support structure. The effect of the pre-tension cable for suppressing unwanted vibrations is illustrated in Figure 4. These results are obtained by finite element dynamic analysis with the excitations generated according to the wind conditions of candidate site. Though a root-mean-square positioning precision of $0.5 \mathrm{~m}$ can be expected for reasonable tension level in the stabilizing cable, it is wise to have a secondary feed stabilizing device instead of increasing the stiffness of the whole structure to an unrealistic level.

To cover the full focal surface of FAST, the maximum length variation of the suspension cable is about 70 meters, compared with about 170 meters in Duan's configuration (Duan et al., 1996). Taking advantage of this, trim masses can be used to balance the static load of the suspension cable for energy efficiency during operation of the telescope. It is relatively easy to drive the cable car because it is driven to move on the suspension cables, instead of directly driving the suspension cables as in Duan's concept (Duan et al., 1996). Parallel suspension and driving cables are arranged for increasing the rotational stiffness of the cable car, see Figure 2.

Compared with an Arecibo-like feed support structure, platformless support configurations have complicated kinematics associated with driving the feed, due to the fact that elastic deformation of the structure has to be considered in positioning the cabin. The proposed cable-car configuration separates the positioning and pointing of the feed, but it uses 7 degrees of freedom to control the 6 degrees of freedom of the feed. Further analytic and experimental research on these aspects is being conducted.

The Stewart platform (Stewart, 1965) has proved to be a high precision approach to controlling the 6 degrees of freedom of a rigid body with high force-to-weight ratio. So the committee of FAST selected the Stewart platform as the secondary stabilizer. This application of the Stewart platform provides a challenge in that the base platform, i.e. the cable car, on which the actuators are mounted, is flexibly 


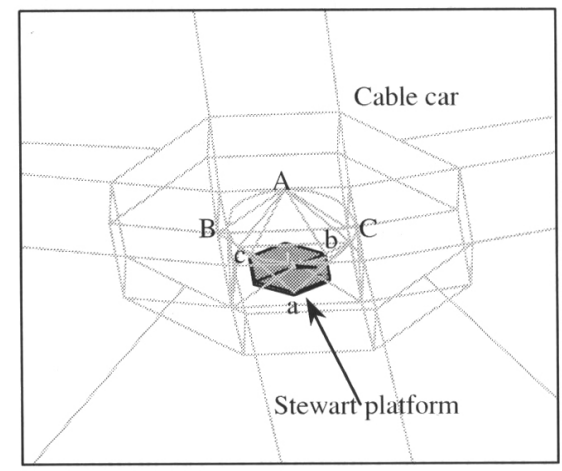

Figure 5. The finite element model of the cable car and the Stewart stabilizer.

TABLE I

The response of the cable car and the stabilized platform

\begin{tabular}{|c|c|c|c|c|c|c|}
\hline \multirow{2}{*}{$\begin{array}{l}\text { Control } \\
\text { updating } \\
\text { freq. }\end{array}$} & \multicolumn{3}{|c|}{ R.M.S. response of the cable car } & \multicolumn{3}{|c|}{ R.M.S. response of the stabilized platform } \\
\hline & Position A & Position B & Position C & Position a & Position $b$ & Position c \\
\hline $10 \mathrm{~Hz}$ & $0.66 \mathrm{~m}$ & $0.50 \mathrm{~m}$ & $0.63 \mathrm{~m}$ & $0.0026 \mathrm{~m}$ & $0.0024 \mathrm{~m}$ & $0.0024 \mathrm{~m}$ \\
\hline $5 \mathrm{~Hz}$ & $0.64 \mathrm{~m}$ & $0.48 \mathrm{~m}$ & $0.61 \mathrm{~m}$ & $0.019 \mathrm{~m}$ & $0.017 \mathrm{~m}$ & $0.017 \mathrm{~m}$ \\
\hline
\end{tabular}

supported. Operation of the actuators will inevitably induce interactions between the base platform and the stabilized feed. This interaction makes the dynamics and control of the system different from most of the cases studied in previous research on Stewart platforms, for which the base platforms are fixed.

A finite element model has been constructed of the cable-car support system with Stewart platform, as shown in Figure 5. Random wind loads have been simulated. A control scheme of difference position prediction is proposed, the effects of which are shown in Table I. Control effects are sensitive to the control updating frequency, and a frequency of $10 \mathrm{~Hz}$ yields the precision required by FAST. Simulations also illustrate that the actuation forces approach that of the static load, for supporting the weight of the feed, when vibration of the feed is suppressed.

\section{Concluding Remarks}

Analyses of the mechanics of the cable car feed support configuration, and simulations of control of the Stewart stabilizer, indicate the feasibility of the proposed support system. An 1:30 scale physical model for the cable-car system, and an 1:5 scale model for the Stewart stabilizer are under construction for further feasibility 
study with the available and proposed technologies. The mapping of cable car movement to the rotations of the driving motors is a key research area for the proposed support configuration.

\section{Acknowledgements}

This work is simultaneously supported by the research fund for large radio telescope, Chinese National Astronomical Observatories, the fundamental research fund (No. JC1999031) of Tsinghua University, and NSFC (19802010). Special thanks are extended to Prof Nan R. for his presentation of this work on behalf of the first author at the conference.

\section{References}

Duan, B., Zhao, Y., Wang, J. and Xu, G.: 1996, Study of the Feed System for a Large Radio Telescope from the Viewpoint of Mechanical and Structural Engineering, in: R.G. Strom, B. Peng and R. Nan (eds.), Proc: of the LTWG-3\&W-SRT, pp. 85-102.

Nan, R., Peng, B., Zhu, W., Zhu, L., Su, Y. and Qiu, Y.: 2000, The FAST Project in China, in: Joe Q. Smith (ed.), Bioastronomy'99 - A New Era in Bioastronomy, Proceedings of a Conference held on the Kohala Coast, Hawaii, 2-6 Aug, 1999, ASP Conference Series 213, 112.

Stewart, D.: 1965, A platform with six degrees of freedom, Proc. Inst. Mech. Eng. 180(5), Part 1, $371-386$. 